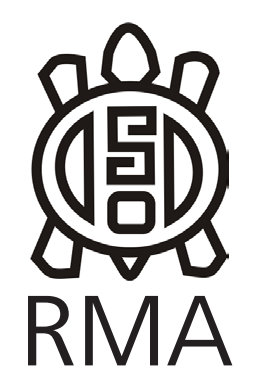

VI Jornadas

Arqueológicas Cuyanas

\title{
Relaciones entre las poblaciones del Valle de Hualfín (dpto. de Belén, prov. de Catamarca) y las situadas al sur. Un caso de estudio a partir de una vasija Sanagasta
}

\author{
Relationships between the populations of Hualfin Valley \\ (Belén, Catamarca province) and those located to the \\ south. A case study from a Sanagasta vessel
}

María Emilia Iucci y Federico Wynveldt*

*Laboratorio de Análisis Cerámico, Facultad de Ciencias Naturales y Museo, Universidad Nacional de La Plata CONICET. E-mail: emiliaiucci@yahoo.com.ar; wynveldtf@fcnym.unlp.edu.ar

\begin{abstract}
Resumen
Los vínculos entre los habitantes del Valle de Hualfín y las poblaciones localizadas al sur han sido mencionados en la bibliografía tradicional, para la época tardía prehispánica y los primeros avances españoles al noroeste, en base a la cerámica y a información de fuentes históricas. En una antigua vivienda de Loma de Ichanga, un sitio tardío de la localidad de La Ciénaga (Valle de Hualfín, Catamarca) fechado en $420 \pm 50$ AP (LP1832), se encontró una vasija Sanagasta, en un contexto que ha sido interpretado como de abandono planificado con incendio, probablemente ritual. El análisis por activación neutrónica y la información petrográfica permitieron interpretar que fue manufacturada con materias primas diferentes a las de las vasijas locales, y por lo tanto postulamos su procedencia en los territorios de influencia Sanagasta, entre el valle de Abaucán (Catamarca) y la provincia de San Juan. Finalmente, sostenemos que la existencia de vínculos entre los habitantes de los momentos tardíos de estas regiones no puede limitarse a movimientos generados por la expansión de una "cultura" o la intervención inkaica, sino que implicaría lazos cultural y temporalmente más profundos.
\end{abstract}

Palabras clave: vasijas Sanagasta; Valle de Hualfín; relaciones interregionales; análisis cerámico; análisis por activación neutrónica.

\begin{abstract}
The links between late inhabitants from Hualfin Valley and populations located at the south were habitually mentioned in bibliography, mainly based in ethnohistoric information, but also in ceramics. In Loma de Ichanga, a late site located in La Ciénaga (Hualfín Valley, Catamarca, Argentina), dated in $420 \pm 50$ BP (LP1832), a Sanagasta vessel was found, in a context that have been interpreted as planned and ritual abandonment with a fire. The neutron activation analysis and the petrographic information led us to establish that the vessel was manufactured with raw materials that are different from those used in local vessels, and let us to think in a foreign origin, probably form Abaucán valley (Catamarca) or La Rioja and San Juan provinces. Finally, we consider deeper cultural and temporal relations between inhabitants than the population politics led by Inka Empire.
\end{abstract}

Keywords: Sanagasta pottery; Hualfín Valley; interregional relationships; ceramic analysis; Neutron Activation Analysis.

La existencia de vínculos de los habitantes tardíos ${ }^{1}$ del Valle de Hualfín con las poblaciones del Valle de Abaucán y las provincias de La Rioja y San Juan fue sugerida históricamente en la literatura arqueológica (e.g. González

\footnotetext{
1 Con esta expresión nos referimos a quienes vivieron durante las épocas conocidas como Período Tardío, Período Inkaico y Período Hispano Indígena.
}

1967), y se encuentra entre las expectativas razonables si se tienen en cuenta las frecuentes prácticas de caravaneo, circulación de bienes y personas en los circuitos conocidos de escala regional, las alianzas y los enfrentamientos en las diferentes coyunturas sociopolíticas.

En las excavaciones de Loma de Ichanga, un sitio tardío 


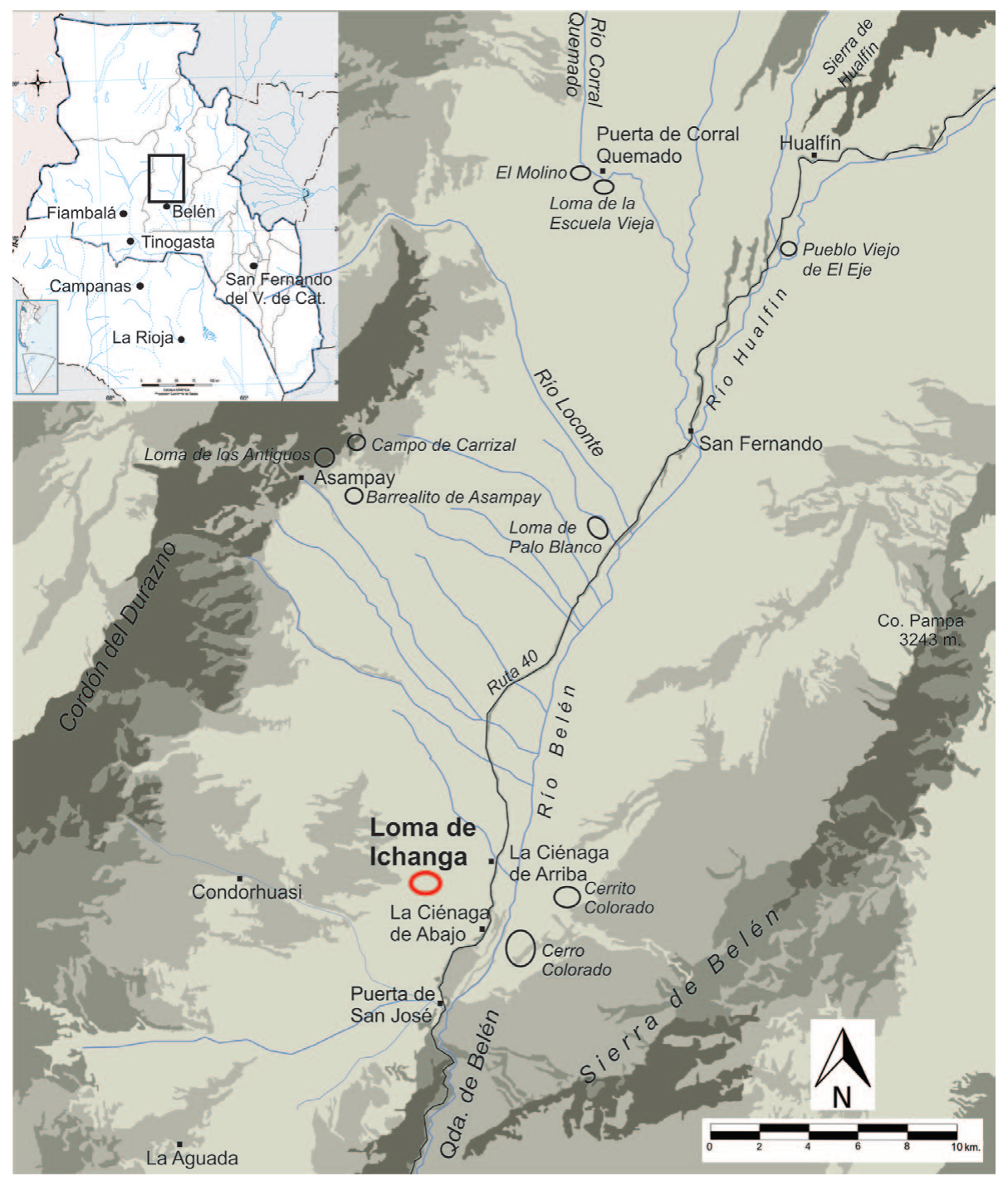

Figura 1. Ubicación del sitio Loma de Ichanga.

Figure 1. Location of Loma de Ichanga site.

los modelos de contacto entre ellas y las dinámicas poblacionales. Para González (1967), Sanagasta era una cultura originada en los valles de Abaucán, Hualfín y Yocavil hacia los años 900-1000 d.C., que habría sido desplazada hacia el sur por parte de las poblaciones Belén y Santa María, con las que habría tenido contactos esporádicos posteriores. Por su parte, Sempé (2005) sostuvo la idea de que Belén era un señorío que se habría expandido y logrado predominar, fusionarse o mantener contactos con las parcialidades de tradición Sanagasta del oeste catamarqueño y de la zona de Andalgalá, sin consolidar un avance más al sur. La llegada de los Inkas habría implicado el traslado de mitimaes Sanagasta al Valle de Hualfín para reemplazar o subordinar a las comunidades Belén.

de La Ciénaga -departamento de Belén, provincia de Catamarca- (Figura 1) se halló una vasija Sanagasta asociada a alfarería Belén, en una estructura que pudo ser utilizada como vivienda. El hallazgo es particularmente relevante porque es uno de los pocos contextos de asociación segura entre ambos tipos de alfarería registrados hasta el momento. En este trabajo se realiza un breve repaso de los resultados obtenidos en estudios previos sobre la vasija Sanagasta de Loma de Ichanga, su contexto de hallazgo y la interpretación del abandono de la estructura donde fue encontrada, con la intención de discutir la índole de las interacciones entre las poblaciones del tardío en la zona.

\section{Las principales líneas interpretativas para las relaciones entre las poblaciones tardías del Valle de Hualfín y las situadas al sur}

Las ideas que se han propuesto tradicionalmente para la interpretación de los vínculos de los habitantes del Valle de Hualfín con las poblaciones de la zona de Abaucán y las provincias de La Rioja y San Juan se relacionan con los supuestos sobre el lugar de origen de las culturas,
Las principales evidencias para presentar estos argumentos fueron el análisis de las fuentes etnohistóricas y la distribución de la alfarería en el terreno. Sin embargo, la diversidad morfológica y decorativa de la cerámica Sanagasta sólo recientemente ha comenzado a revisarse en profundidad (e.g. Sempé y García 2002; Revuelta et al. 2010-2011) y, en lo que al Valle de Hualfín respecta, su importante variabilidad plantea una dificultad adicional a su estado fragmentario predominante, de tal modo que algunos elementos del estilo no son claramente discernibles de los de otras alfarerías de la zona. Por otro lado, más allá de las numerosas piezas enteras que forman parte de los depósitos de museos, son muy pocos los contextos de hallazgo documentados con vasijas Sanagasta. Uno de estos contextos es el entierro de Barrealito de Asampay (Sempé et al. 1995-1996), que fue interpretado como intrusivo, no se asocia a otros elementos contemporáneos y no tiene datación absoluta. Esta situación no difiere significativamente por fuera del Valle de Hualfín, en donde existen pocos contextos de asociación segura entre piezas Sanagasta y Belén, como el entierro de Campanas (Revuelta 2009) y el de La Troya. Este último fue fechado en $501 \pm 29,566 \pm 34$ y $603 \pm$ 
37 (MTC-15596, AA 92176 y AA 62809 respectivamente) (Ratto 2013), y puede considerarse coincidente con algunas de las dataciones más tempranas de los sitios tardíos del Valle de Hualfín (Wynveldt y lucci 2013).

Más allá de estos casos, las evidencias arqueológicas atribuibles a vínculos entre los habitantes del Valle de Hualfín y las poblaciones de la zona sur del Noroeste y de la zona norte del Centro-Oeste argentino no han sido sustanciales y quedaron subordinadas a las también escasas referencias de las fuentes escritas.

\section{La olla Sanagasta de Loma de Ichanga y su contexto}

Loma de Ichanga es un pequeño poblado antiguo ubicado sobre una lomada de unos $50 \mathrm{~m}$ de altura del piedemonte del cordón occidental de las sierras del Valle de Hualfín (Figura 1). Las estructuras arqueológicas corresponden a 15 recintos cuadrangulares de piedra. Se excavaron dos recintos completos (6 y 9) y una trinchera (Recinto 7), y se hallaron restos de cerámica Belén y, en el Recinto 9, cerámica ordinaria, ambos tipos alfareros predominantes en los sitios de la zona.

En el Recinto 6, de $14 \mathrm{~m}^{2}$, se excavaron $80 \mathrm{~cm}$ de profundidad, con un único nivel de ocupación. En el centro de la estructura se registraron dos hoyos para poste y, a pocos centímetros, una tinaja Belén completamente fragmentada que pudo reconstruirse casi en su totalidad. Asociados a ella, un fragmento de un hueso largo parcialmente calcinado y una figurina de cerámica, consistente en un rollo de arcilla con un esbozo de piernas, cabeza y cabello que le dan una semblanza humana. En el ángulo oeste del recinto se encontró una concentración de marlos carbonizados y, algo alejada de las paredes, la vasija Sanagasta fracturada, que yacía sobre el piso y contenía en su interior restos de marlos de maíz. En el piso de la estructura no había otros materiales -como vasijas fracturadas $u$ otros restos de alimentos- que pudieran interpretarse como resultado de descartes frecuentes durante las actividades cotidianas del grupo

Figura 2. Arriba: Fotografía y dibujo de la vasija Sanagasta. Abajo: Diagrama de dispersión de los Componentes Principales 1 y 2 de las mediciones por activación neutrónica, clasificados por tipo cerámico $(\mathrm{N}=73)$, aplicando el criterio selectivo. En la esquina superior izquierda del diagrama se encuentra la vasija Sanagasta de Loma de Ichanga.

Figure 2. Up: Photography and draw of the Sanagasta vessel. Bottom: Principal component scatter plot (Component 1 and 2 ) of the neutron activation measurements, sorted by ceramic type $(\mathrm{N}=73)$. Selective criteria applied. Loma de Ichanga's Sanagasta vessel is shown in the upper left corner of the plot. humano que lo habitaba. Esta ausencia ha conducido a plantear la hipótesis de que se trataba de una vivienda que era frecuentemente limpiada, o al menos lo habría sido inmediatamente antes de su abandono (Balesta y Wynveldt 2010; lucci 2013; Valencia et al. 2010).

Sobre estos materiales se hallaron los restos carbonizados de las estructuras de sostén y enramada del techo. No se encontraron restos del poste central -cuya existencia está indicada por la presencia de los hoyos del centro de la estructura-, lo cual dio lugar a la consideración de que habría sido retirado con anterioridad al incendio que ocasionó la carbonización del techo. Junto con las evidencias de incendios de otras estructuras residenciales de varios sitios del Valle de Hualfín, estos indicadores fueron considerados como indicios de un abandono planificado y ritual del recinto (Balesta y Wynveldt 2010; Valencia et al. 2010).

El fechado radiocarbónico efectuado sobre uno de los marlos de maíz (Balesta y Wynveldt 2010) dio como resultado $420 \pm 50$ AP (LP1832). La calibración para un sigma (68,2\% de probabilidad) dio un rango entre 14491510 AD (42,6\%) y $1578-1621$ AD $(25,6 \%)$, y para dos sigmas un rango entre 1443-1629 AD $(95,4 \%)$ utilizando la curva SHCal13 (Hogg et al. 2013). Esta fecha ha permitido a sugerir que el abandono pudo haber tenido relación con el impacto generado por la conquista inkaica entre los grupos locales (Wynveldt y Balesta 2010).

\section{Caracterización de la vasija Sanagasta}

La vasija que clasificamos como Sanagasta (Figura 2) es
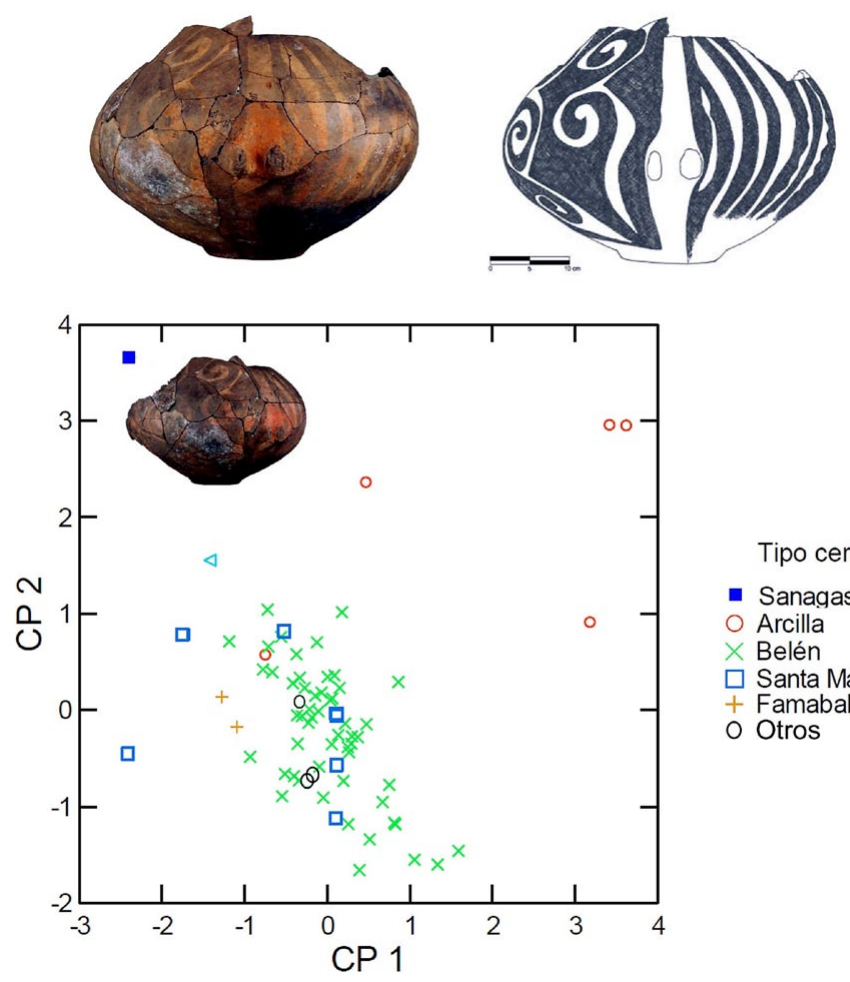

Tipo cerámico

- Sanagasta

Arcilla

$\times$ Belén

$\square$ Santa María

+ FamabalastoNG

O Otros 
restringida y de forma elipsoide. Su diámetro mayor (de 39 $\mathrm{cm}$ ) se ubica a $15,8 \mathrm{~cm}$ de la base. Tiene al menos un asa -la otra no fue encontrada-, una base cóncavo-convexa de $12,5 \mathrm{~cm}$ de diámetro y una leve restricción que demarcaba un cuello corto. Sus fragmentos se reconstruyeron en un solo bloque que forma alrededor del $40 \%$ de la vasija.

La superficie externa es alisada y pintada en negro sobre un fondo color crema, y en algunos sectores la pintura está desleída y puede observarse la coloración rojiza de la superficie cerámica. El dibujo forma dos paneles, delimitados por la posición de las asas. Uno de ellos presenta volutas, mientras que el otro líneas rectas y festoneadas paralelas. Tanto la distribución de los paneles como las imágenes pintadas en su interior corresponden a las características del estilo según ha sido descrito por Revuelta y colaboradores (2010-2011). La superficie interna es alisada, pero sin pintura.

La vasija presenta desgaste dado por el uso en la porción inferior de la pared interna y en el sector de apoyo externo. La distribución del hollín depositado sobre ambas caras sugiere que éste fue producto de un fuego posterior a la ruptura. La caída del techo habría generado que la porción de vasija dejada en la estructura se rompiera en varios fragmentos y se tiznara. El hecho de que tuviera pequeños restos de marlos de maíz en su interior revela que aunque estuviera rota probablemente todavía podía ser usada a modo de contenedor (Iucci 2013).

Se analizó un fragmento de la vasija por petrografía y por activación neutrónica, con la finalidad de comparar su composición con la cerámica Belén y ordinaria, consideradas de manufactura local. En términos petrográficos, siguiendo los criterios enunciados en un trabajo previo (lucci 2013) se observó que la pasta es de color entre castaño oscuro y castaño medio, con orientación regular, las cavidades son de forma irregular y la matriz es arcillo-limosa con inclusiones félsicas (cuarzo y feldespatos) y micas (biotita) en la fracción de limo observable en el microscopio. La proporción matriz-cavidades-inclusiones es 68-6-26\%, y entre los componentes de tamaño arena se destaca el cuarzo (54\% de las inclusiones) y los litoclastos plutónicos y sedimentarios (26\%). Además, se observaron muy escasos fragmentos pumíceos y litoclastos volcánicos. En su conjunto, estas proporciones se diferencian notoriamente de las de las pastas Belén (con arenas con mayor diversidad de elementos componentes) y ordinaria (con tiesto molido y arenas de composición variada) (lucci 2013).

El análisis por activación neutrónica, presentado previamente (lucci 2013), se realizó sobre una muestra de 68 fragmentos de distintos tipos cerámicos (54 Belén, 7 Santa María, el único fragmento Sanagasta, y 6 de otros tipos tardíos) de varios sitios del Valle, y 5 muestras de arcillas (cuatro de la localidad de Asampay, cocidas; y una de La Ciénaga, no cocida). Sobre las mediciones realizadas por el Grupo de Trabajo Técnicas Analíticas Nucleares del Centro Atómico de Ezeiza se llevó a cabo estadística básica, la estandarización logarítmica de la matriz de datos y un análisis por componentes principales (ACP) con matriz de covarianza y rotación Varimax (Iucci 2013).

La estadística básica muestra que la vasija Sanagasta presenta la mayor parte de sus elementos por fuera del primer desvío estándar, e incluso los valores de muchos de ellos son outliers (Tabla 1). En relación al ACP se realizaron dos ensayos. En el análisis de criterio selectivo se seleccionaron los elementos con valor de incertidumbre analítica menor al 15\%, que sumaron 11 en total: Ce, Eu, La, Lu, Sm y Yb (tierras raras); Cs (elemento alcalino), Sc, Co y Fe (elemento de transición); y Th (actínido). EI ACP generó $11 \mathrm{CP}$, entre los cuales los primeros dos explican un 81,848\% de la variación ( $C P 1=57,791+C P 2=24,058)$. Los pesos de los componentes cayeron mayormente en las tierras raras La, Ce, Eu y Sm para el CP1, y en el alcalino Cs para el CP2 (Tabla 2).

En el ensayo de criterio no selectivo se tuvieron en cuenta todos los elementos medidos con la excepción de As y Gd que no fueron determinados para todas las muestras, totalizando 19 elementos: 7 tierras raras (Ce, Eu, La, $\mathrm{Lu}, \mathrm{Sm}, \mathrm{Tb}$ y $\mathrm{Yb}), 3$ alcalinos ( $\mathrm{Ba}, \mathrm{Cs}, \mathrm{Rb}), 6$ metales de transición (Sc, Hf, Co, Cr, Fe y Ta), 2 actínidos (Th y U), y 1 metaloide (Sb). La suma de los CP1 $(46,752 \%)$ y CP2 $(16,276 \%)$ explicó el $63,028 \%$ de la variación. El CP3 $(11,458 \%)$ aportó una variación poco menor al CP2. Para el CP1 los pesos de los elementos cayeron en los mismos que en el CP1 del primer ensayo, en el CP2 el elemento que aportó mayor variación fue el alcalino Cs, pero también inciden el alcalino $\mathrm{Rb}$, el metaloide $\mathrm{Sb}$ y el actínido U. Para el CP3 aportaron variabilidad los metales Cr, Fe y Co (Tabla 2).

La correlación de ambos CP para el primer (Figura 2) y el segundo ensayo produjo una nube conformada por la gran mayoría de las muestras. La vasija Sanagasta es la pieza cerámica que más se aleja del grupo central, y a su vez se separa de las arcillas de Asampay, que también quedan por fuera del grupo central. Ante el peso importante del Cesio en el CP2, se realizó una correlación entre el CP1 y el CP3 con los elementos medidos en el segundo ensayo, con el resultado de que la vasija Sanagasta queda igualmente apartada del resto de los fragmentos cerámicos ${ }^{2}$. Observados en conjunto, los

\footnotetext{
2 Se realizaron grupos de semejanza química mediante análisis de conglomerados jerárquicos, corregidos por análisis discriminante y distancia de Mahalanobis (lucci 2013). Además se llevaron a cabo análisis de componentes principales extrayendo algunos elementos como el Cs -que en uno de los componentes explicaba buena parte de la variabilidad-, y los otros elementos alcalinos, y se realizaron pruebas excluyendo las arcillas; con el fin de observar si eran determinantes para la diferenciación de la vasija Sanagasta respecto del resto de la muestra. Todos los resultados, que no se exhiben aquí por razones de espacio, fueron coherentes en el apartamiento de esta vasija y coincidieron en que son varios los elementos que contribuyen a la
} 


\begin{tabular}{cccccccc}
\hline Elemento & Min & Max & 11.3 & X & DS & X+DS & X-DS \\
\hline $\mathrm{Ba}$ & 325 & 630 & 370 & 514,8 & 104,3 & 619,1 & 410,5 \\
$\mathrm{Ce}$ & 58,8 & 124,9 & $\mathbf{5 8 , 8}$ & 89,3 & 17,8 & 107,0 & 71,5 \\
$\mathrm{Co}$ & 8,49 & 16,52 & $\underline{\mathbf{8}, 49}$ & 14,5 & 2,0 & 16,5 & 12,5 \\
$\mathrm{Cr}$ & 28,2 & 47,9 & 30,7 & 38,7 & 10,8 & 49,4 & 27,9 \\
$\mathrm{Cs}$ & 9,2 & 45,9 & $\underline{45,9}$ & 17,7 & 10,4 & 28,1 & 7,3 \\
$\mathrm{Eu}$ & 1 & 1,75 & $\underline{1}$ & 1,5 & 0,2 & 1,7 & 1,3 \\
$\mathrm{Fe}$ & 25130 & 46060 & $\underline{25130}$ & 40327,0 & 4852,8 & 45179,8 & 35474,2 \\
$\mathrm{Hf}$ & 3,54 & 6,17 & 4,24 & 5,2 & 2,0 & 7,1 & 3,2 \\
$\mathrm{La}$ & 32,77 & 54,75 & 32,77 & 44,6 & 8,5 & 53,1 & 36,1 \\
$\mathrm{Lu}$ & 0,062 & 0,694 & 0,563 & 0,5 & 0,1 & 0,6 & 0,4 \\
$\mathrm{Rb}$ & 136 & 339 & $\underline{339}$ & 183,9 & 35,6 & 219,5 & 148,3 \\
$\mathrm{Sb}$ & 0,551 & 3,16 & 1,84 & 1,4 & 0,8 & 2,2 & 0,5 \\
$\mathrm{Sc}$ & 10,55 & 17,52 & $\underline{10,55}$ & 15,0 & 2,0 & 17,0 & 13,0 \\
$\mathrm{Sm}$ & 4,54 & 10,79 & 6,44 & 8,4 & 1,7 & 10,2 & 6,7 \\
$\mathrm{Ta}$ & 1,25 & 2,5 & 1,44 & 1,6 & 0,3 & 1,9 & 1,3 \\
$\mathrm{~Tb}$ & 0,66 & 1,6 & 1,31 & 1,1 & 0,3 & 1,3 & 0,8 \\
$\mathrm{Th}$ & 11,76 & 29,5 & $\underline{11,76}$ & 16,9 & 2,7 & 19,6 & 14,2 \\
$\mathrm{U}$ & 3,27 & 13 & $\underline{13}$ & 5,3 & 1,6 & 6,9 & 3,7 \\
$\mathrm{Yb}$ & 2,43 & 3,97 & 2,69 & 3,1 & 0,7 & 3,7 & 2,4 \\
\hline
\end{tabular}

Tabla 1. Principales estadísticos de la muestra y valores obtenidos para la vasija Sanagasta. Min: valor mínimo de la muestra; Max: valor máximo de la muestra; 11.3 : vasija Sanagasta; $X=$ Promedio; $D S$ : desvío estándar. Los valores outliers se calcularon en base al rango intercuartil. En negrita se destacan los elementos que caen por fuera del DS1 y en negrita subrayados aquellos considerados outliers.

Table 1. Sample main statistics and resulting values from Sanagasta vessel. Min; minimal value of the sample; Max: maximum value of the sample; 11.3 Sanagasta vessel; $\mathrm{X}=$ mean; DS: standard deviation. The outliers were calculated on interquartile range. The elements positioned outside DS1 are marked in bold. In bold and underline are marked the outliers.

\begin{tabular}{|c|c|c|c|c|c|c|}
\hline \multicolumn{3}{|c|}{ Análisis con criterio selectivo } & \multicolumn{4}{|c|}{ Análisis con criterio no selectivo } \\
\hline & $\mathrm{CP} 1$ & $\mathrm{CP} 2$ & & CP 1 & CP 2 & $\mathrm{CP} 3$ \\
\hline $\log C e$ & 0,922 & 0,193 & $\log L a$ & 0,916 & 0,138 & 0,167 \\
\hline $\log \mathrm{Eu}$ & 0,894 & 0,215 & $\log C e$ & 0,909 & 0,009 & 0,205 \\
\hline $\log L a$ & 0,873 & 0,311 & $\log \mathrm{Eu}$ & 0,858 & 0,034 & 0,313 \\
\hline $\log S m$ & 0,804 & 0,371 & $\log S m$ & 0,847 & 0,199 & 0,134 \\
\hline $\log T h$ & 0,717 & 0,194 & $\log Y b$ & 0,762 & 0,324 & 0,149 \\
\hline $\log S c$ & 0,676 & 0,537 & $\log T h$ & 0,734 & 0,039 & 0,073 \\
\hline $\log Y b$ & 0,656 & 0,466 & $\log T b$ & 0,694 & 0,145 & $-0,165$ \\
\hline $\log \mathrm{Fe}$ & 0,619 & 0,233 & $\log \mathrm{Hf}$ & 0,679 & 0,565 & 0,036 \\
\hline $\log L u$ & 0,504 & 0,513 & $\log L u$ & 0,632 & 0,405 & 0,041 \\
\hline $\log C o$ & 0,455 & 0,033 & $\log S c$ & 0,595 & 0,393 & 0,589 \\
\hline \multirow[t]{9}{*}{$\log C s$} & 0,059 & 0,997 & $\log \mathrm{Fe}$ & 0,396 & 0,111 & 0,75 \\
\hline & & & $\log C s$ & 0,246 & 0,944 & 0,09 \\
\hline & & & $\log S b$ & 0,24 & 0,741 & 0,409 \\
\hline & & & $\log R b$ & 0,182 & 0,761 & 0,124 \\
\hline & & & $\log C o$ & 0,182 & $-0,057$ & 0,705 \\
\hline & & & $\log B a$ & 0,175 & $-0,096$ & 0,169 \\
\hline & & & $\log U$ & 0,062 & 0,74 & $-0,404$ \\
\hline & & & $\log T a$ & $-0,072$ & 0,197 & $-0,207$ \\
\hline & & & $\log C r$ & $-0,14$ & 0,099 & 0,922 \\
\hline
\end{tabular}

resultados son significativos porque indican que la pieza Sanagasta se diferencia notablemente en su composición del resto de las muestras, y permite sostener su origen como no local. En el caso de las piezas Santa María, Famabalasto Negro Grabado y de otros tipos tardíos no-Belén, los resultados no fueron contundentes como para postular con certeza una diferencia con la cerámica Belén (Iucci 2013).

\section{Conclusiones}

El foco de interés puesto en este trabajo sobre una vasija particular tiene como propósito la apertura de algunas vías para discutir problemáticas relacionadas con las interacciones interregionales durante el tardío, que hasta el momento no se han basado en evidencias arqueológicas claras. En el caso de la vasija de Loma de Ichanga, encontramos una correspondencia general de forma y decoración con las piezas caracterizadas como Sanagasta. El análisis petrográfico nos

Tabla 2. Matriz de componentes rotados, según el análisis selectivo y no selectivo.

diferenciación de esta muestra del resto del conjunto. 
permitió diferenciar su pasta de la de las vasijas tardías manufacturadas en el Valle de Hualfín, mientras que el resultado del análisis por activación neutrónica es consecuente con la idea de que se trata de una vasija de manufactura no local. En síntesis, esta vasija pudo haber sido elaborada en algún taller alfarero con tradición de manufactura Sanagasta, posiblemente en las zonas de Abaucán (provincia de Catamarca), La Rioja o San Juan. Posteriormente, pudo haber sido llevada desde su lugar de manufactura hacia el Valle de Hualfín, no a causa de un traslado poblacional -traslados para los que hasta el momento no registramos evidencias arqueológicas concretas en la zona-, sino en un contexto de frecuentes intercambios de bienes y de circulación de personas. Allí fue incorporada a las prácticas de uso corrientes de las vasijas cerámicas del valle.

La modalidad de depósito del recinto nos llevó a sugerir, en trabajos previos, un abandono planificado y ritual. En este contexto, tanto la tinaja Belén, que se hallaba entera con anterioridad a la caída del techo, como la figurina de cerámica y la olla Sanagasta, que aún fracturada conservaba las características visuales del estilo y su potencialidad de uso como contenedor, posiblemente cargaban con las connotaciones simbólicas otorgadas por quienes habían habitado el lugar, y pudieron haber sido dejadas intencionalmente allí como parte del abandono.

Finalmente, si bien se han planteado alternativas para la interpretación de las relaciones entre las poblaciones Belén y Sanagasta (e.g. Ratto 2013), consideramos que los vínculos entre los habitantes de los momentos tardíos en estas regiones no se limitaron a movimientos generados por la expansión de una "cultura" o por la intervención inkaica, sino que implicaban lazos cultural y temporalmente más profundos. Desde este punto de vista, el caso de la vasija Sanagasta de Loma de Ichanga representa, quizás, más la continuidad en las interacciones grupales largamente sostenidas en el tiempo, durante un momento de cambios sociopolíticos devenidos con la presencia inkaica hacia el siglo XV d.C., que la presencia de hipotéticos mitimaes trasladados al Valle de Hualfín por los Inkas como manifestación de su política imperial.

Berisso, 15 de febrero de 2016

\section{Agradecimientos}

Al Equipo de Técnicas Analíticas Nucleares del Centro Atómico Nacional de Ezeiza por la realización del Análisis de Activación Neutrónica. Al Lic. Martín Morosi por la colaboración en la observación petrográfica del corte delgado. A las Lics. Claudia Cavarozzi y Claudia Di Lello por la colaboración en la interpretación geoquímica. A los evaluadores del manuscrito que contribuyeron a darle mayor claridad al texto. No obstante, todo lo expresado aquí es de exclusiva responsabilidad de los autores.

\section{Bibliografía}

Balesta, B. \& F. Wynveldt. 2010. Defensibilidad, visibilidad y abandono en la Loma de Ichanga del Valle de Hualfín (Depto. de Belén, Prov. de Catamarca, Argentina). Revista Española de Antropología Americana 40(1): 53-71.

González, A. R. 1967. Una excepcional pieza del mosaico cultural del N. O. argentino. Consideraciones sobre la secuencia arqueológica de la Prov. de San Juan. Etnía 6.

Hogg, A, Q. Hua, P. Blackwell, M. Niu, C. Buck, T. Guilderson, T. Heaton, J. Palmer, P. Reimer, R. Reimer, C. Turney \& S. Zimmerman. 2013. SHCal13 Southern Hemisphere Calibration, 0-50,000 years cal BP. Radiocarbon 55(4): 1889-1903.

lucci, M. E. 2013. "Producción, circulación y uso de cerámica tardía del Valle de Hualfín. Catamarca, Argentina". Facultad de Ciencias Naturales y Museo, Universidad Nacional de La Plata, Argentina: 572 p. Tesis de Doctorado. SEDICI (Repositorio Institucional de la UNLP) http://sedici.unlp.edu.ar/ handle/10915/35235.

Ratto, N. 2013. A modo de introducción: la articulación de estudios arqueológicos, paleoambientales e históricos en el oeste tinogasteño (Catamarca). N. Ratto, Delineando prácticas de la gente del pasado: Los procesos socio-históricos del oeste catamarqueño, Introducción, 17-44. Sociedad Argentina de Antropología, Buenos Aires.

Revuelta, C. 2009. Informe material arqueológico Campanas. M.S.

Revuelta, C. M., S. A. Carosio \& J. P. Aguilar. 2010-2011. Formas y representaciones tardías. Aproximaciones a una mirada integral al estilo cerámico Sanagasta - Angualasto. Anales de Arqueología y Etnología 65-66: 57-85.

Sempé, M. C. 2005. El Período Tardío en Azampay: el señorío Belén y su modelo geopolítico. Sempé, M. C., S. Salceda y M. Maffia. Azampay. Presente y pasado de un pueblito catamarqueño, 365-380. Ediciones Al Margen, La Plata.

Sempé, M. C. \& D. García. 2002. Relación y continuidad estilística de los grupos cerámicos Sanagasta-San José. Shincal 7: 1-8.

Sempé, C., G. Méndez \& S. Salceda. 1995-1996. Entierro intrusivo en Barrealito de Azampay. Shincal 5: 43-49.

Valencia, M. C; M. Fernández \& C. Barberis. 2010. Evidencias de Incendios en el registro arqueológico de La Ciénaga. B. Balesta y N. Zagorodny, Aldeas protegidas, conflicto y abandono. Investigaciones arqueológicas en La Ciénaga (Catamarca), capítulo 4, 161-199. Ediciones Al Margen, La Plata.

Wynveldt, F. \& M. E. lucci. 2013. La cronología Belén en el norte del Valle de Hualfín: viejos problemas, nuevas perspectivas. Revista Arqueología 19(1): 187-195. 